\title{
A NOTE ON STRONG LOCALLY DIVIDED DOMAINS
}

\author{
By
}

\author{
David E. DoBBs
}

\begin{abstract}
A uniform proof is given for the following five assertions. Let $R$ be an integral domain such each overring of $R$ is a pseudovaluation domain (resp., divided domain; resp., going-down domain; resp., locally pseudovaluation domain; resp., locally divided domain). Then $R / P$ has the same property, for each prime ideal $P$ of $R$. The assertion for pseudovaluation domains was proved recently by Okabe-Yoshida by other methods.
\end{abstract}

\section{Introduction and summary.}

Let $R$ be a pseudovaluation domain (for short, PVD), in the sense of [7]; that is, $(R, M)$ is a quasilocal (commutative integral) domain with a canonically associated valuation overring $V$ such that $M$ is the maximal ideal of $V$. In [7, Proposition 2.7], Hedstrom-Houston showed that each overring of $R$ (including $R$ itself) is a PVD if and only if $V$ is the integral closure of $R$. Recently, Okabe-Yoshida have given other equivalent characterizations [8, Theorem 2.4], and have shown that the property in question is stable under passage to factor domains [8, Proposition 2.5]. In this note, we use uniform methods to prove [8, Proposition 2.5] and four new related results.

Following [8], if $\boldsymbol{P}$ is a property of domains, we may say that a domain $R$ is strong $\mathbb{P}$ in case each overring of $R$ satisfies $\mathbb{P}$. With this terminology, [8, Proposition 2.5] asserts that if $R$ is a strong PVD, then so is $R / P$, for each prime ideal $P$ of $R$. This is recovered in Theorem 2.2(a) below. In Theorem $2.2(\mathrm{~b})$, (c), we prove the analogue for divided domains and going-down domains. (Recall from [1] and [3] that a domain $R$ is called divided in case $P R_{P}=P$ for each prime ideal $P$ of $R$. Each PVD is a divided domain [4, page 560]; and each divided domain is a going-down domain [3, Proposition 2.1], in the sense of [2].) In Theorem 2.2(d), (e), we establish the analogues for locally pseudovaluation domains (or LPVD's, in the sense of [6]) and locally divided domains (in the sense of [5]).

Received January 9, 1990. 
Any unexplained material is standard, as in the cited articles.

\section{Results.}

We begin by isolating two assertions that will be needed in the proof of parts (d) and (e) of Theorem 2.2 .

LEMMA 2.1. Let $P$ be a prime ideal of an LPVD (resp., a locally divided domain) $R$. Then $R / P$ is an LPVD (resp., a locally divided domain).

ProOF. Put $S=(R / P)_{Q / P}$, where $P \subset Q$ are prime ideais of $R$. It suffices to show that $S$ is a PVD (resp., divided domain). Since $S$ is canonically isomorphic to $R_{Q} / P R_{Q}$ and $R_{Q}$ is a PVD (resp., divided domain), it suffices to observe that the class of PVD's (resp., divided domains) is stable under passage to factor domains. For this, one may appeal to [4, Lemma 4.5(i)] (resp., [3, Lemma 2.2(c)]). The proof is complete.

Theorem 2.2(a) recovers [8, Proposition 2.5]. In view of the many inequivalent usages of "strong" in the literature, we prefer to state this result without the "strong" terminology.

THEOREM 2.2. Let $P$ be a prime ideal of a domain $R$. Then:

(a) If each overring of $R$ is a PVD, then each overring of $R / P$ is a PVD.

(b) If each overring of $R$ is a divided domain, then each overring of $R / P$ is a divided domain.

(c) If each overring of $R$ is a going-down domain, then each overring of $R / P$ is a going-down domain.

(d) If each overring of $R$ is an LPVD, then each overring of $R / P$ is an LPVD.

(e) If each overring of $R$ is a locally divided domain, then each overring of $R / P$ is a locally divided domain.

Proof. Consider an overring $E$ of $R / P$. Thus $R / P \subset E \subset R_{P} / R P_{P}$. Put $T=R+P R_{P}$. One has a canonical isomorphism $T / P R_{P} \cong R / P$. Thus, by a standard homomorphism theorem, $E \cong D / P R_{P}$ where $D$ is a domain satisfying $T \subset D \subset R_{P}$. In particular, $D$ is an overring of $R$. By hypothesis, $D$ is a PVD (resp., divided; resp., a going-down domain; resp., an LPVD; resp., locally divided). It suffices to observe that the class of PVD's (resp., divided domains; resp., going-down domains; resp., LPVD's; resp., locally divided domains) is stable under passage to factor domains. For this, one may appeal to [4, Lemma 
4.5(i)], [3, Lemma 2.2(c) and Remark 2.11], and Lemma 2.1. The proof is complete.

REMARK 2.3. (a) Perhaps the simplest example of a domain $R$ satisfying the conditions in Theorem 2.2 is $F-X K[[X]]$, where $F \subset K$ is an algebraic field extension.

(b) The absence of an assertion about "locally going-down domains" in Theorem 2.2 is explained by the fact that a domain $R$ is a going-down domain if and only if $R_{P}$ is a going-down domain for each prime ideal $P$ of $R[3$, page 357]. In particular, a domain satisfying any of the conditions in Theorem 2.2 must be a going-down domain. However, not all going-down domains (or PVD's) satisfy those conditions: cf. [2, Theorem 4.2(ii)].

(c) The unified proof in Theorem 2.2 becomes especially simple in part (a) and (b). (Recall that (a) recovered the motivating result, [8, Proposition 2.5].) Indeed, in these cases, $T=R$ since $R$ is divided, and so the proof amounts just to the isomorphism $E \cong D / P$ and an appeal to the stability results ([4, Lemma 4.5(i)], [3, Lemma 2.2(c)]).

(d) The interested reader may find more complicated proofs of Theorem 2.2 by using pullbacks and various results in the literature on seminormality, $i$-domains, and Prüfer domains. One such proof uses [6, Theorem 2.9]. In this regard, we close by noting that [6, Corollary 2.10$]$ provides some characterizations of strong PVD's, and thus may be viewed as a companion for $[8$, Theorem 2.4].

\section{References}

[1] T. Akiba, A note on AV-domains, Bull. Kyoto Univ. Education Ser. B, 31 (1967), 1-3.

[2] D.E. Dobbs, On going down for simple overrings, II, Comm. Algebra, I (1974), $439-458$.

[3] D. E. Dobbs, Divided rings and going-down, Pac. J. Math., 67 (1976), 353-363.

[4] D.E. Dobbs, Coherence, ascent of going-down, and pseudo-valuation domains, Houston J. Math., 4 (1978), 551-567.

[5] D.E. Dobbs, On locally divided integral domains and CPI-overrings, Internat. J. Math. \& Math. Sci., 4 (1981), 119-135.

[6] D.E. Dobbs and M. Fontana, Locally pseudo-valuation domains, Annali Mat. Pura Appl., 134 (1983), 147-168.

[7] J.R. Hedstrom and E. G. Houston, Pseudo-valuation domains, II, Houston J. Math., 4 (1978), 199-207.

[8] A. Okabe and K. Yoshida, Note on strong pseudo-valuation domains, Bull. Fac. Sci. Ibaraki Univ. Ser. A, 21 (1989), 9-12.

Department of Mathematics

University of Tennessee

Knoxville, Tennessee 37996-1300

U.S.A. 